\title{
Review
}

\section{Transferrin isoforms in cerebrospinal fluid and their relation to neurological diseases}

\author{
By Yuta Murakami, ${ }^{* 1}$ Kiyoshi SAito, ${ }^{* 1}$ Hiromi ITO $^{* 2}$ and Yasuhiro Hashimoto ${ }^{* 3, \dagger}$
}

(Communicated by Kunihiko SuZUKI, M.J.A.)

\begin{abstract}
Iron plays many important roles in the brain, including involvement in myelination, neurotransmission and electron transfer in the respiratory chain. Transferrin (Tf), an iron transporter, is mainly biosynthesized in the liver, but can also be biosynthesized in the brain; i.e., by oligodendrocytes and the choroid plexus, a cerebrospinal fluid (CSF) producing tissue. The CSF contains two Tf isoforms, brain-type Tf and serum-type Tf, which differ in their glycan structures. Brain-type Tf is uniquely glycolsylated with biantennary asialo- and agalacto-complex type $N$-glycans that carry bisecting $\beta 1,4-G l c N A c$ and core $\alpha 1,6-F u c$. The glycans of serum-type Tf in the CSF are similar to those of Tf in serum. Biochemical analyses reveal that the apparent molecular size of brain-type $\mathrm{Tf}$ is smaller than that of serum-type Tf, and that hydrophobic patches are exposed on brain-type $\mathrm{Tf}$ as demonstrated by hydrophobic probe binding studies. We found that brain-type Tf levels were decreased in idiopathic normal pressure hydrocephalus, in which CSF production is suspected to decrease, while brain-type Tf increased in spontaneous intracranial hypotension, in which CSF production is suspected to increase. These results suggest that braintype Tf could be a biomarker of altered CSF production.
\end{abstract}

Keywords: biomarker, cerebrospinal fluid, transferrin, lipocalin-type prostaglandin D synthase, intracranial hypotension syndrome, idiopathic normal pressure hydrocephalus

\section{Introduction}

Iron is an essential nutrient for physiological processes throughout the body, including the brain. Oligodendrocytes require iron for myelination and store large amounts of ferritin-bound iron. In

\footnotetext{
*1 Department of Neurosurgery, School of Medicine, Fukushima Medical University, Fukushima, Japan.

*2 Department of Biochemistry, School of Medicine, Fukushima Medical University, Fukushima, Japan.

*3 Department of Human Life Science, School of Nursing, Fukushima Medical University, Fukushima, Japan.

$\dagger$ Correspondence should be addressed: Y. Hashimoto, Department of Human Life Science, School of Nursing, Fukushima Medical University, 1 Hikarigaoka, Fukushima, Fukushima 9601295, Japan (e-mail: yasuc@fmu.ac.jp).

Abbreviations: APP: amyloid precursor protein; CNS: central nervous system; CP: choroid plexus; CSF: cerebrospinal fluid; FAB: frontal assessment battery; Ig: immunoglobulin; iNPH: idiopathic normal pressure hydrocephalus; L-PGDS: lipocalin-type prostaglandin D synthase; MMSE: mini-mental state examination RI: radioisotope; sAPP: soluble amyloid precursor protein; SDSPAGE: sodium dodecyl sulfate-polyacrylamide gel electrophoresis; SIH: spontaneous intracranial hypotension; Tf: transferrin; TTR: transthyretin.
}

contrast, astrocytes retain less iron. ${ }^{1}$ As a multipurpose nutrient, iron is involved in oxygen transport, electron transport and many other fundamental physiological processes. ${ }^{2)}$ However, free iron results in cellular toxicity by generating harmful reactive oxygen species (ROS). ${ }^{3)}$ Iron, therefore, needs to bind to specific carrier proteins such as transferrin (Tf) in the extracellular fluid and blood.4),5) Tf is a single polypeptide chain with 679 amino acid residues, consisting of two homologous $\mathrm{N}$ - and C-terminal domains. ${ }^{6)}$ Each domain has a metal binding pocket with high affinities for ferric iron, which are crucial for preventing ROS generation and subsequent cell damage.

Central nervous system (CNS) iron is mainly derived from blood Tf. $^{7)}$ However, the CNS is separated from the systemic circulation by the blood-brain barrier (BBB), which is comprised of endothelial cells, basement membrane, pericytes and astrocytic processes. Blood Tf binds to transferrin receptor 1 (TfR1) on endothelial cells lining blood capillaries and the binding complex is internalized 


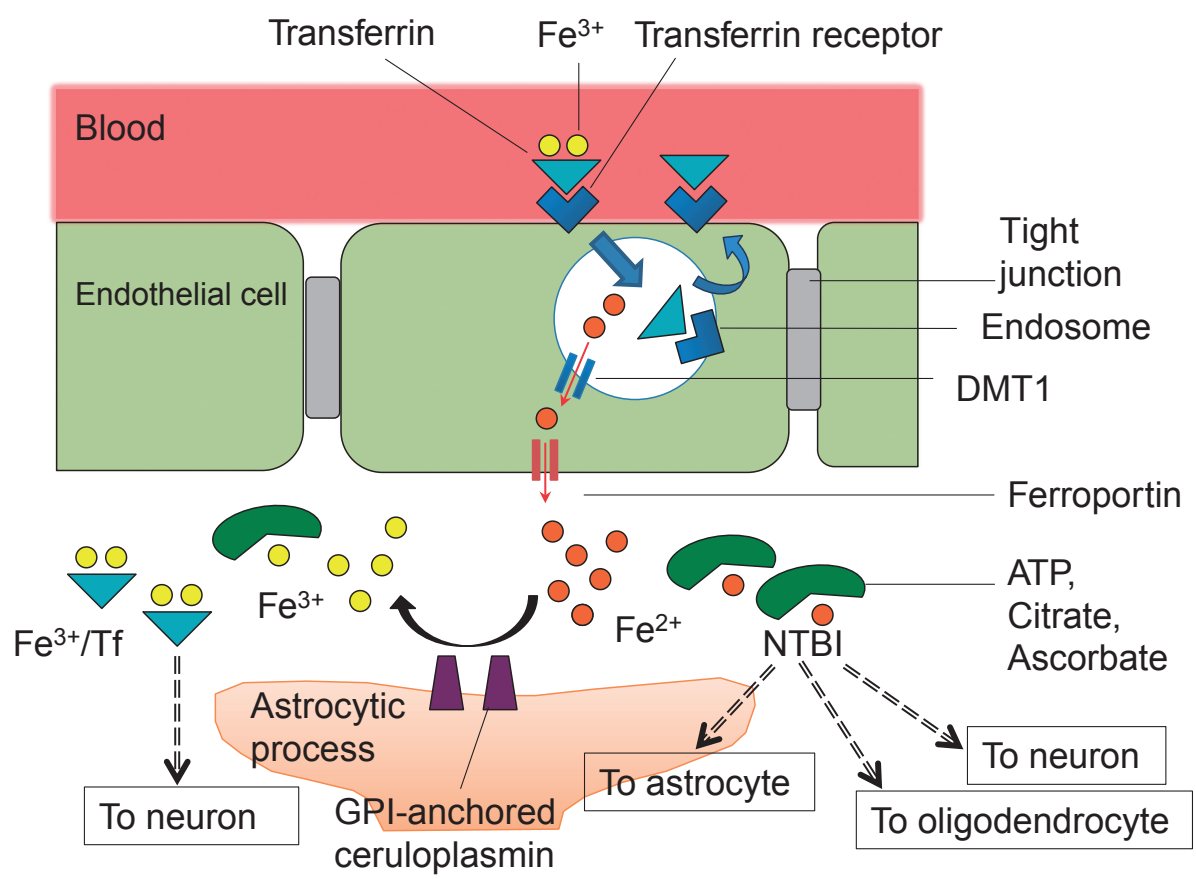

Fig. 1. Iron transport into the brain via blood-brain barrier. Transferrin $(\mathrm{Tf})$ carries ferric iron $\left(\mathrm{Fe}^{3+}\right)$ in the blood. The Tf complex $\left(\mathrm{Fe}^{3+} / \mathrm{Tf}\right)$ binds to the transferrin receptor (TfR1) on the luminal membrane of endothelial cells and is subsequently internalized into endosomes where the acidic $\mathrm{pH}$ causes $\mathrm{Fe}^{3+}$ to be released from the complex (TfR1/ $\mathrm{Tf} / \mathrm{Fe}^{3+}$ ). The released $\mathrm{Fe}^{3+}$ is reduced to ferrous iron $\left(\mathrm{Fe}^{2+}\right)$ by endosomal reductases and then transported in the cytosol by divalent metal transporter 1 (DMT1). It is then exported to the abluminal side by ferroportin. The exported $\mathrm{Fe}^{2+}$ is oxidized to $\mathrm{Fe}^{3+}$ by GPI-anchored ceruloplasmin on astrocytic process. $\mathrm{Fe}^{3+}$ binds to $\mathrm{Tf}$ in the interstitial fluid ( $\mathrm{Fe}^{3+} / \mathrm{Tf}$ ), which is taken up by neuron. In the interstitial fluid, some $\mathrm{Fe}^{2+}$ binds to ATP, citrate, and ascorbic acid (non-transferrin-bound iron; NTBI).

into endosomes (Fig. 1). ${ }^{2)}$ After internalization of the $\mathrm{Tf} / \mathrm{TfR} 1$ complex, the iron dissociates from Tf in an acidic endosomal compartment. The iron-free transferrin (apotransferrin) together with TfR1 is then transported back to the plasma membrane where it is released into the blood to participate in further rounds of iron mobilization and delivery. Ferric iron $\left(\mathrm{Fe}^{3+}\right)$ released from Tf is reduced to ferrous ion $\left(\mathrm{Fe}^{2+}\right)$ by endosomal reductase, with the $\mathrm{Fe}^{2+}$ then transported across the endosomal membrane into the cytosol by the divalent metal transporter, DMT1.4) The $\mathrm{Fe}^{2+}$ is further exported to the abluminal side by ferroportin. The exported $\mathrm{Fe}^{2+}$ is oxidized to $\mathrm{Fe}^{3+}$ by the astrocytic ferroxidase, glycosylphosphatidylinositol (GPI)-anchored cerulopasmin. Parenchymal $\mathrm{Fe}^{3+}$ immediately binds to Tf, thereby preventing ROS generation. In this way, $\mathrm{Fe}^{3+}$ carried by blood $\mathrm{Tf}$ is transported to parenchymal Tf via endothelial cells (Fig. 1).

Neurons express high levels of TfR 1 , which binds the Tf $/ \mathrm{Fe}^{3+}$ complex to be internalized. After internalization, the iron is released and either stored as ferritin-bound iron or passed into the mitochondria, where iron is incorporated into haem and ironsulphur clusters. There are two possible pathways that Tf can follow after the internalization; one is "recycling" in the brain interstitial fluid, as is the case for blood Tf, and the other is degradation in neurons. It remains to be clarified, however, which pathway is dominant in the neuron. In contrast to neurons, oligodendrocytes and astrocytes take up iron as nontransferrin-bound iron (NTBI), in which $\mathrm{Fe}^{2+}$ binds to ATP, citric acid and ascorbic acid (Fig. 1).

It was demonstrated that $\mathrm{Tf}$ is biosynthesized by neural cells such as oligodendrocytes and CSFproducing epithelial cells in the choroid plexus. Blay et al. reported that Tf mRNA is expressed in choroid plexus epithelium, ${ }^{8)}$ while De Arriba Zerpa et al. reported that $\mathrm{Tf}$ mRNA in rat primary cultured oligodendrocytes is spliced and lacks the N-terminal signal sequence. These findings suggest that $\mathrm{Tf}$ proteins produced by oligodendrocytes reside in the cytosol and are not secreted. ${ }^{9)}$ 
A

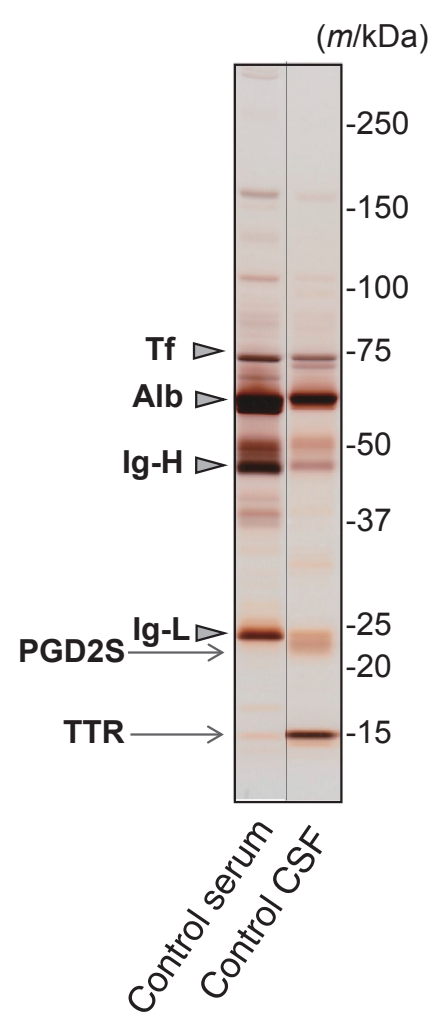

B

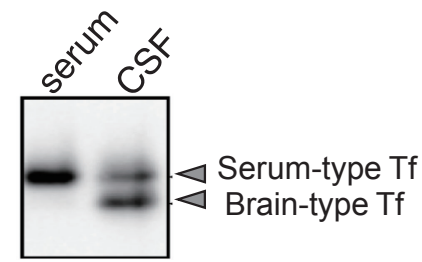

Immunoblotting by anti-Tf Ab
C

Hydranencephaly cases

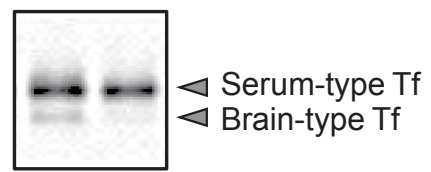

Immunoblotting by anti-Tf Ab

D
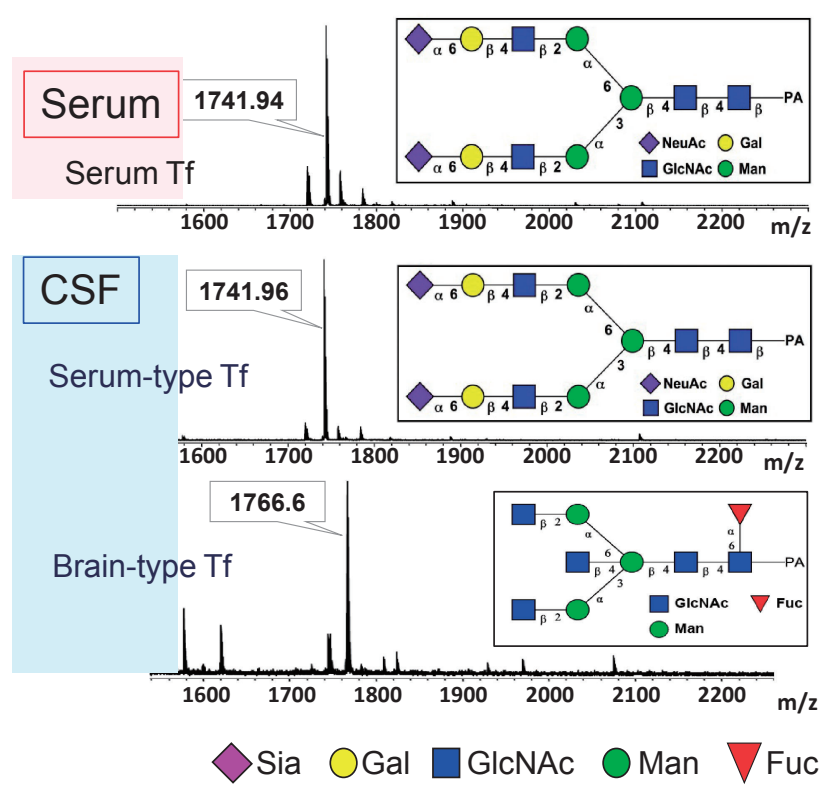

Fig. 2. CSF protein analysis. Human CSF and serum were analyzed by SDS-PAGE followed by silver staining (A) or immunoblotting using an anti-Tf antibody (B, C). Migrating positions of serum transferrin (Tf), albumin (Alb), immunoglobulin heavy chain (Ig-H), and light chain (Ig-L) are indicated with arrowheads (A). Bands for lipocalin-type prostaglandin D synthetase (L-PGDS) and transthyretin (TTR) are indicated with arrows. The positions of brain- and serum-type Tf are indicated with arrowheads on immunoblots for control CSF (B) and CSF-like fluid from a hydranencephaly patient (C). Matrix-assisted laser desorption/ionizationtime of flight (MALDI-TOF) mass spectrometry (MS) spectra of $N$-glycans derived from serum, and serum-, and brain-type transferrin (D). The major peak of brain-type Tf glycan is at $m / z$ 1766.6. The major peak was subjected to tandem MS analysis and its fragmented patterns were identical to those of the authentic glycan; its schematic structure is shown in the lowest inset. The upper inset shows the glycan structure of sialylated serum transferrin. $N$-glycans derived from serum and serum-type Tf were desialylated and subjected to MALDI-TOF-MS analysis. Both $N$-glycans show major peaks around $m / z$ 1741.9. Sugar symbols are used according to the Consortium for Functional Glycomics for the representation of glycan structures.

\section{Transferrin and its glycan isoform in cerebrospinal fluid (CSF)}

When the protein compositions of human serum and cerebrospinal fluid (CSF) are compared using sodium dodecyl sulfate-polyacrylamide gel electrophoresis (SDS-PAGE) followed by silver staining, the major CSF protein bands are similar to those in serum except for the added presence of lipocalin-type prostaglandin D synthase (L-PGDS) and transthyretin (TTR) (Fig. 2A). The result is consistent with a previous report showing that $80 \%$ of CSF proteins are derived from the serum. ${ }^{10)}$ The specimens were also examined by Western blot analysis using antibodies against serum glycoproteins with a view to detecting CSF-specific isoforms. Indeed, Western blot analysis using anti-Tf antibody detected two isoforms in CSF: one with a similar migration position to serum $\mathrm{Tf}$ and the other with a faster migration position (Fig. 2B). This latter isoform is named "brain-type" Tf, also designated as asialo- Tf or Tf-1, while the former isoform is named serum-type 
Tf sialo-Tf or Tf-2. ${ }^{11)-13)}$ The concentrations of braintype and serum-type Tf, quantified by chromatoscanning Western blots, were $8.0 \pm 3.9 \mu \mathrm{g} / \mathrm{mL}$ and $18.6 \pm 5.6 \mu \mathrm{g} / \mathrm{mL}$, respectively $(\mathrm{n}=10)$.

Migration speed differences can often be detected for different glycan isoforms carried by the same protein. To analyze the glycan structures of purified Tf isoforms, the glycans were liberated by $N$ glycosidase $\mathrm{F}$ treatment and derivatized to pyridylamino (PA)-glycans for mass spectrometry (MS) analysis. MS analysis of the PA-glycans revealed a major peak for brain-type Tf glycan at $m / z$ 1766.6. Tandem mass spectrometry analysis of the major peak revealed a fragmented pattern that was similar to that of authentic biantennary asialo- and agalactocomplex type $N$-glycans that carry bisecting $\beta 1,4-$ GlcNAc and core $\alpha 1,6$-Fuc (Fig. 2D, lower panel). In contrast, glycans from serum-type Tf were similar to those of serum transferrin, i.e., MS analysis revealed that the major peaks of the desialylated PA-glycans from serum-type $\mathrm{Tf}$ and those from serum transferrin were at $m / z 1741.96$ and 1741.94, respectively (Fig. 2D, middle and upper panels). Glycan structures of serum, serum-type, and braintype Tf were further analyzed by lectin microarray, which revealed the presence of $\alpha 2,6$-sialyl residues on serum-type Tf.

Serum-type Tf has sialyl $\alpha 2,6$ galactosyl $\beta 1,4 N$ acetylglucosamine (GlcNAc) at its non-reducing end whereas brain-type $\mathrm{Tf}$ has a truncated or GlcNAc-terminated glycan, which is a brain-type glycan. The truncation is speculated to be due to the low expression of $\beta 1,4$ galactosyltransferase in the brain. ${ }^{14)}$ Nakakita et al. reported that brain-type glycan accounts for $5.3 \%$ of total $N$-glycans in the mouse brain, ${ }^{15)}$ indicating it is specific to the brain. Indeed, the glycan is carried by several brain-derived proteins such as prostaglandin D synthetase ${ }^{16)}$ and $\beta$ secretase (or BACE1). ${ }^{13)}$

\section{Effect of glycan structure on physicochemical properties and functions of transferrin}

Glycosylation may stabilize proteins by providing a surface cover consisting of intramolecular glycan-polypeptide interactions and possibly inducing a conformational change in the polypeptide, resulting in glycan-dependent molecular properties and functions. ${ }^{17)}$ To test this hypothesis, we subjected Tf isoforms to comparative biochemical analyses and found that the apparent molecular size (hydration volume) of brain-type Tf is smaller than that of serum-type $\mathrm{Tf}$ based on size exclusion chromatography (Fig. 3A). ${ }^{18)}$ Fluorescence correlation spectrometry analysis revealed that the hydrodynamic radius of brain-type Tf was smaller than of serum Tf, which was consistent with the sizeexclusion chromatography data. These results suggested that the conformation of brain-type $\mathrm{Tf}$ is changed by a unique glycan. The isoform was, therefore, subjected to X-ray crystallographic analysis; however, the conformation of the polypeptide main chain (data not shown) was very similar to that of a previously reported Tf structure, ${ }^{19)} \mathrm{PDB}$ code 3V83. We subsequently examined the surface properties of isoforms using the fluorescent probe ANS (1-anilino-8-naphthalenesulfonate), which has been widely used to monitor transitions in proteins owing to its affinity for partially exposed hydrophobic regions. ${ }^{20)}$ Addition of ANS to brain-type Tf induced a marked enhancement of fluorescence, ${ }^{18)}$ with a maximum at $492 \mathrm{~nm}$ (Fig. 3C). In contrast, ANS shows only weak fluorescence with serum $\mathrm{Tf}$ at $504 \mathrm{~nm}$. ANS alone shows weak fluorescence at $508 \mathrm{~nm}$. The strong, red-shifted fluorescence of ANS in the presence of brain-type Tf suggests that it binds to an exposed hydrophobic surface or pocket in this Tf isoform.

Next, we examined functional differences in $\mathrm{Tf}$ isoforms. The most evident function of Tf is its high affinity binding to ferric iron. Equilibrium dialysis analysis using ${ }^{59} \mathrm{Fe}$ revealed that the isoforms did not show any apparent difference in iron binding affinity (unpublished data). The isoforms were further examined for their binding to TfR1, which was stably expressed on $\mathrm{CHO}$ cells. Again, the isoforms did not show any difference in binding affinity for the receptor (unpublished data). Although Tf glycans influence physicochemical properties such as the hydration volume and exposure of hydrophobic patches, they did not affect any of the functions we examined. Further analyses are required to identify potential functional differences between the isoforms.

\section{Histochemistry using anti-Tf antibody and lectins}

To examine the origin of brain-type Tf, we analyzed the CSF of patients with hydranencephaly, a congenital malformation of the brain. In hydranencephaly, the cerebral hemispheres are absent due to bilateral occlusion of the internal carotid arteries, whereas the midbrain, cerebellum and other brain structures nourished by the vertebral artery may remain. ${ }^{21), 22)}$ Brain-type Tf was decreased markedly in the CSF of hydranencephaly patients, indicating 
A

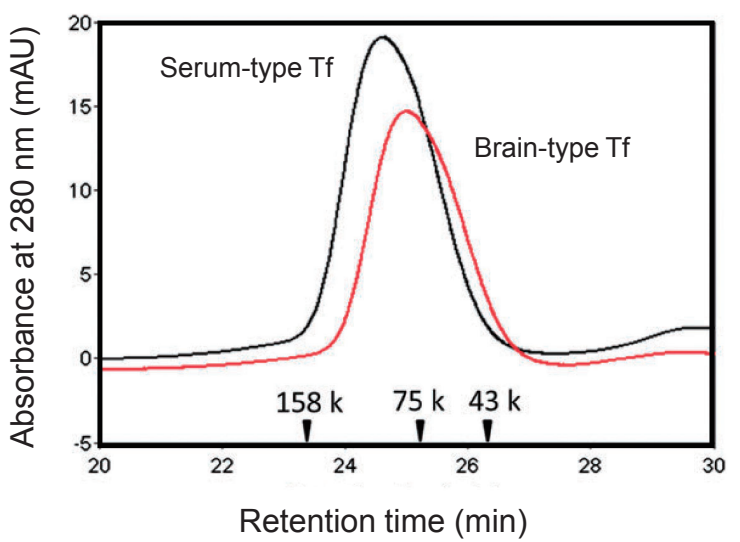

C

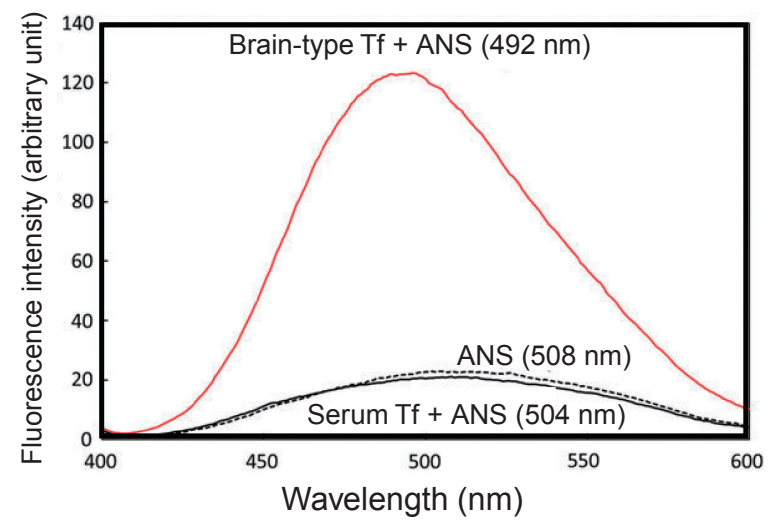

B

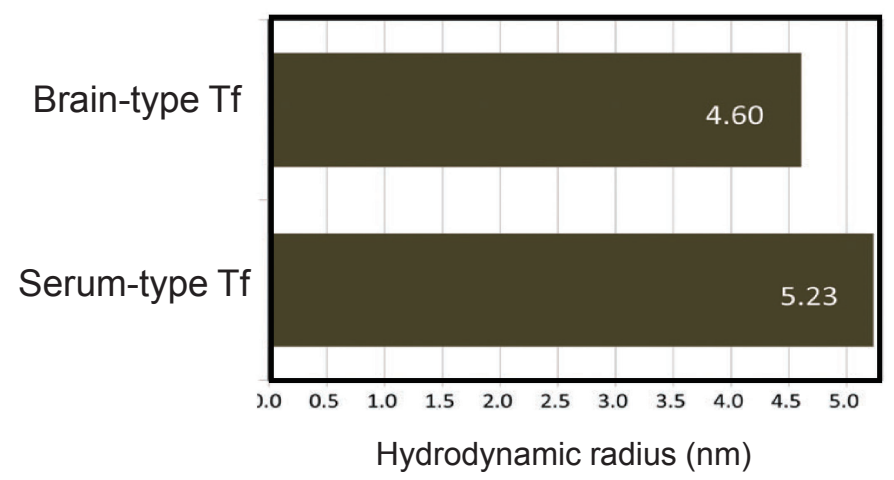

Fig. 3. Physicochemical analysis of Tf isoforms. Estimation of molecular size of serum- and brain-type Tf by size-exclusion chromatography (A). The retention time of standard proteins (aldolase, $158 \mathrm{kDa}, 23.4 \mathrm{~min}$; conalbumin, $75 \mathrm{kDa}, 25.2 \mathrm{~min}$; ovalbumin, $43 \mathrm{kDa}, 26.4 \mathrm{~min}$ ) is indicated with black triangles. Fluorescence correlation spectroscopy (FCS) analysis of Tf isoforms (B). FCS shows Stokes-Einstein hydrodynamic radius of serum-type and brain-type Tf. Fluorescence spectra of 1-anilino-8-naphthalene sulfonate (ANS) binding assay for Tf isoforms (C). ANS alone (black solid line), presence of brain-type Tf (red line) or serum Tf (black dotted line). Protein and ANS concentrations were 2 and $140 \mu \mathrm{M}$, respectively. The excitation wavelength was set at $390 \mathrm{~nm}$.

that brain-type $\mathrm{Tf}$ is derived from the cerebrum (Fig. 2C). ${ }^{23)}$

We next examined the localization of $\mathrm{Tf}$ in the human cerebrum. Immunohistochemistry using antiTf antibodies revealed that the most intense signal was detected in the choroid plexus epithelium (Fig. 4A, B). Perls' staining to detect iron also revealed strong signals in the choroid plexus epithelium (Fig. 4C), suggesting that holo-Tf (iron-saturated form of $\mathrm{Tf}$ ) is formed in the epithelium and secreted into the CSF. To analyze glycan(s) expressed by the epithelium, the choroid plexus was stained with two types of lectin; one was Psathyrella velutina lectin (PVL), which is specific to GlcNActerminated glycan; ${ }^{24}$ ) and the other was Sambucus sieboldiana agglutinin (SSA), which is specific to $\alpha 2,6$-sialyl-terminated glycan. The choroid plexus was first co-stained with PVL lectin and Tf-antibody, with the latter staining portions of the choroid plexus epithelium, interstitial tissues and capillary blood clots (Fig. 4E, left panel). High magnification imaging showed preferential staining of basolateral aspect of the epithelium, with minimal staining of apical aspect (Fig. 4E, right panel). The basolateral signals partially overlapped with those of PVL signals (Fig. 4G, right panel). Other areas were minimally stained with PVL lectin, suggesting that GlcNActerminated glycoproteins are localized specifically in the epithelium (Fig. 4F). Next, the choroid plexus was co-stained with SSA lectin and anti-Tf antibody. SSA strongly stained some interstitial tissues and blood clots (Fig. 4J, left panel), with SSA staining 


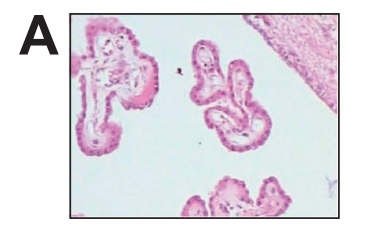

H.E. staining
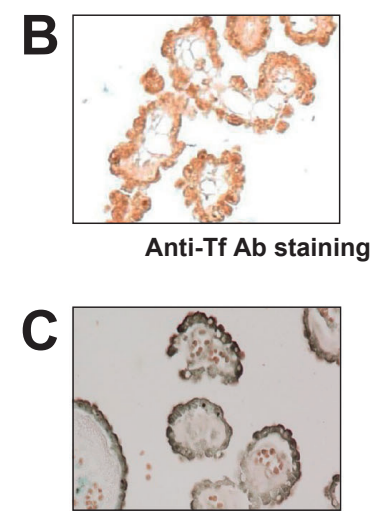

Iron-staining
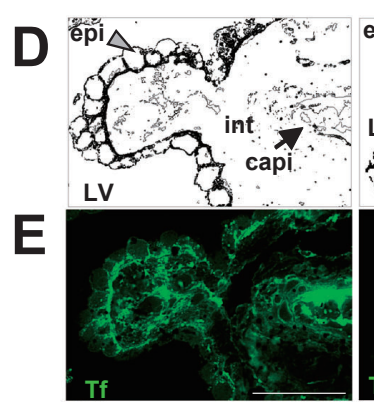

$\mathbf{F}$

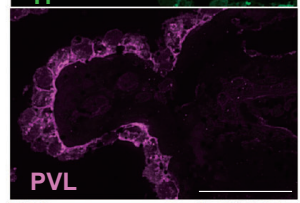

G

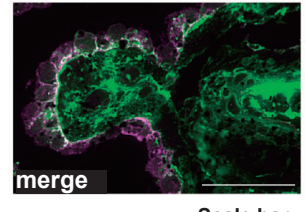

Scale bar:

$50 \mu \mathrm{m}$
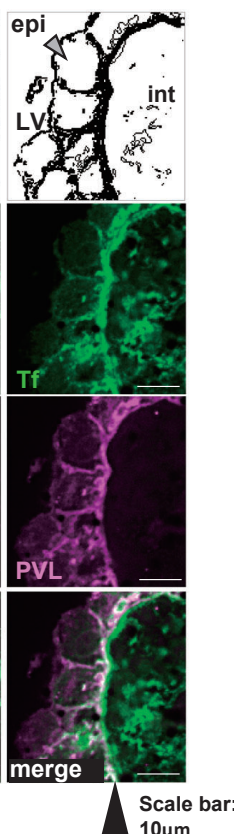
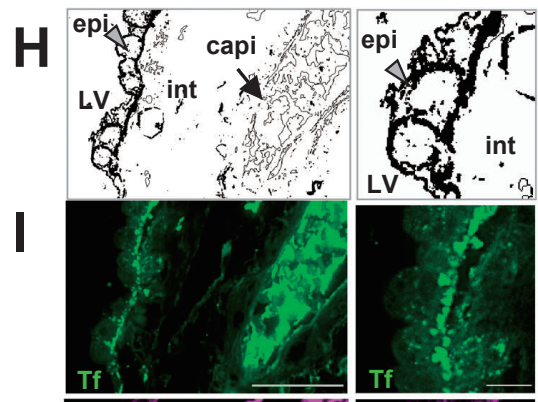

J
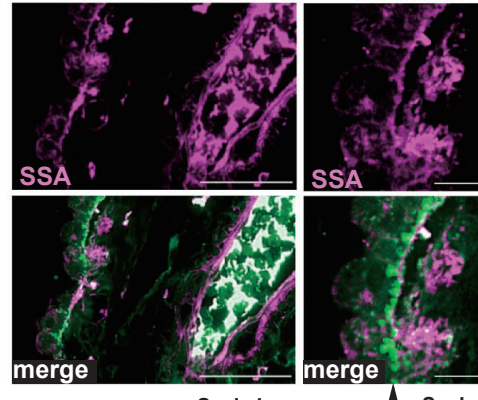

K

Scale bar:

$50 \mu \mathrm{m}$

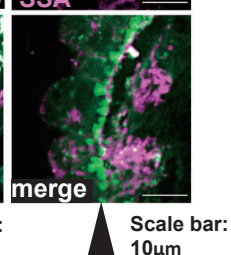

Fig. 4. Histochemical analysis of the choroid plexus. Human choroid plexus slices were stained with Hematoxylin-Eosin (A), anti-Tf antibody (B), and Perls' Prussian blue dye for iron staining (C). Illustrations D and H are schematic representations of the choroid plexus; choroid plexus epithelial cells (epi), interstitial tissue (int), blood capillaries (capi), and lateral ventricle (LV). In E-K, choroid plexus tissue slices were stained with anti-Tf antibody (E, I), PVL lectin (F), and SSA lectin (J). The anti-Tf antibody signal was merged with that of PVL $(\mathrm{G})$ or SSA lectin $(\mathrm{K})$. For each set of images, a higher magnification of the left panel is shown in the right panel.

substantially overlapping with strong anti-Tf antibody positivity (Fig. 4K, left panel). In contrast, the majority of SSA staining on basolateral portions of the epithelium did not overlap that of $\mathrm{Tf}$ antibody (Fig. 4K, right panel). These results suggest that the epithelium specifically expresses GlcNAc-terminated glycoproteins in choroid plexus tissue and possibly produces brain-type Tf. $^{23)}$

\section{Changes in brain-type transferrin in neurological disease}

Idiopathic normal pressure hydrocephalus (iNPH). Patients with idiopathic normal pressure hydrocephalus (iNPH) have excess intracranial CSF, which compresses the brain parenchyma. Resulting in cognitive impairment, gait disturbance, and urinary incontinence among the major symptoms. In iNPH, excess CSF may decrease further CSF production. ${ }^{25)}$ At least two CSF biomarkers have been identified for iNPH. These include L-PGDS, which is mainly produced by the leptomeninges, arachnoid membrane, and choroid plexus, ${ }^{26)}$ while soluble amyloid precursor protein ( $\mathrm{SAPP}$ ) is secreted by neurons. ${ }^{27)}$ L-PGDS and SAPP are decreased in the CSF of iNPH patients, ${ }^{28), 29)}$ which fits with a previous report by us. ${ }^{12)}$ Brain-type Tf levels are decreased in the CSF of iNPH patients (Fig. 5A, B). The concentration of brain-type $\mathrm{Tf}$ in the CSF was quantified by chromatoscanning as shown in Fig. 5C. Patients with suspected iNPH can be further diagnosed by lumbar puncture or continuous lumbar drainage, in which excess CSF is temporally removed as a means to improvement symptoms. In continuous lumbar drainage, the patient undergoes continuous withdrawal of CSF for 6 days. Compared to day 0 , the $\mathrm{Tf}$ isoform concentration increased from day $1-3 \quad(126 \pm 11 \%$, not significant $)$ and more conclusively from day $4-6 \quad(143 \pm 17 \%, p<0.01)$ $(\mathrm{n}=4)$, indicating that brain-type Tf production began to increase within a week of drainage onset (Fig. 5D). Data on serum-type Tf is not included in Fig. 5 because the lumbar drainage procedure may result in contamination with blood $\mathrm{Tf}$, the concentration of which is $40-50$-fold higher than that of CSF.

The long-term recovery of brain-type Tf was analyzed after shunt surgery for hydrocephalus. The isoform is markedly increased during post-operative 
A

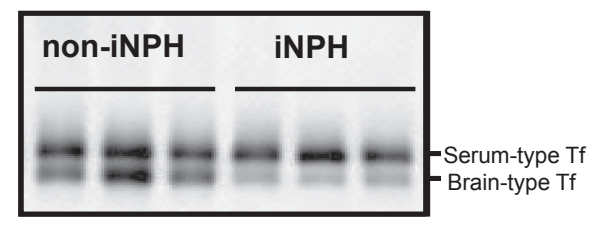

D

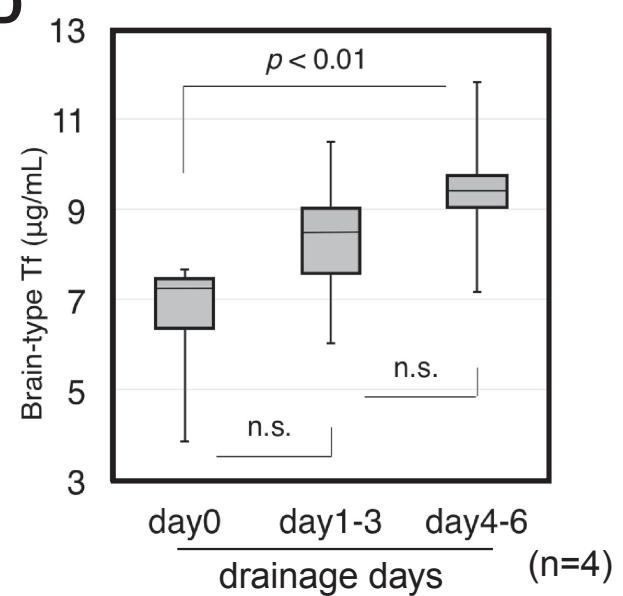

B

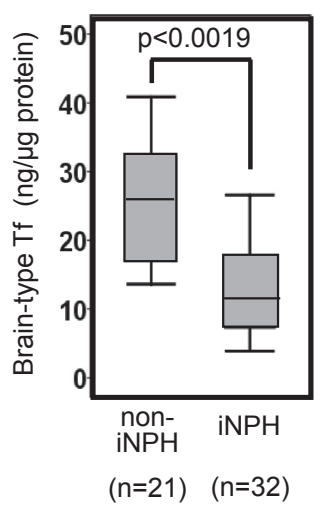

C

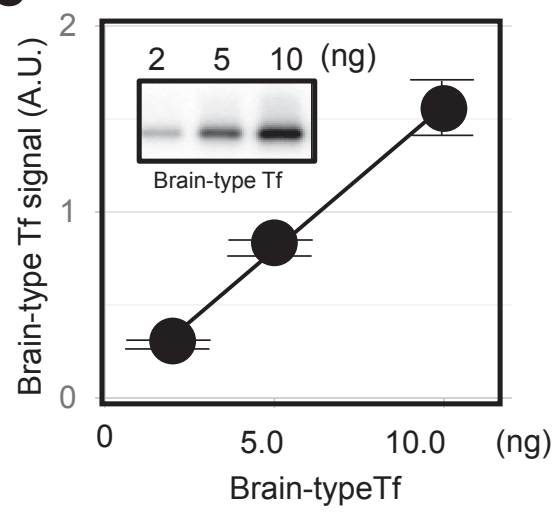

E

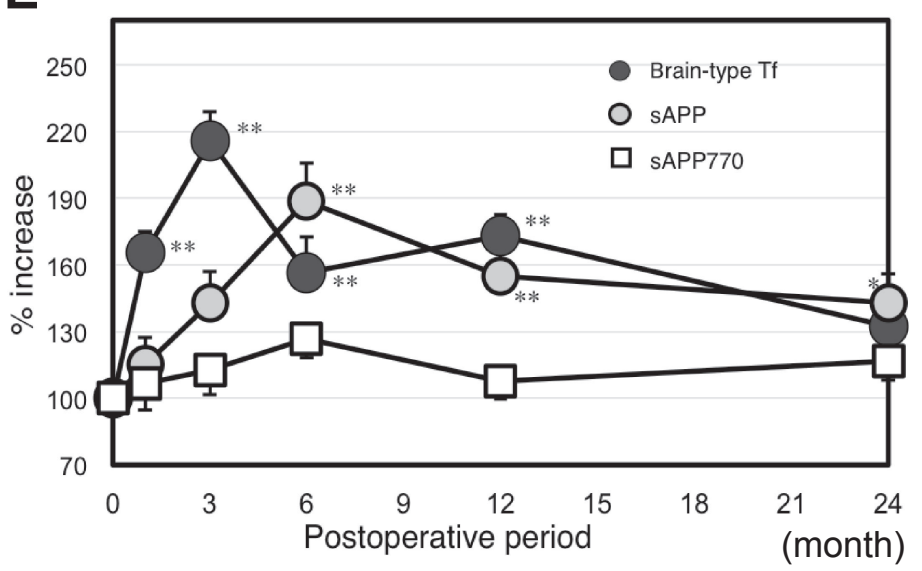

Fig. 5. Concentration of brain-type Tf in the CSF of iNPH patients. Brain-type Tf decreases in the CSF of iNPH patients compared to that of non-iNPH patients (A, B). The concentration of brain-type Tf was quantified by chromatoscanning using the calibration curve (C). Significant differences were found (Steel-Dwass tests $(p<0.0019)$ ) $(\mathrm{C})$. For iNPH diagnosis, some patients underwent continuous CSF drainage for 6 days to examine improvement of symptoms. Brain-type Tf levels before drainage and post-drainage days 1-3 or 4-6 are indicated with box plots $(n=4)$ (D). Significant differences were identified (Dunnett's multiple comparison analysis, $p<0.01$ ); n.s., not significant. For curative intervention, iNPH patients underwent shunt surgery, which bypasses excess CSF from brain's ventricles to the peritoneal cavity. CSF biomarker levels were quantified pre-surgery (indicated as $100 \%$ at month 0 ) and postsurgery (mean \pm S.E.); brain-type Tf (filled black circles), soluble form of amyloid precursor protein (sAPP) (filled gray circles), and its isoform sAPP770 (open squares). Significant differences were found (Dunnett's multiple comparison analysis) and are indicated by asterisks; $p<0.05(*), p<0.01(* *)$.

months $1-3$ and then gradually declines from months 6-24, suggesting that brain-type $\mathrm{Tf}$ is an indicator for the recovery of CSF production (Fig. 5E). ${ }^{30)} \mathrm{In}$ contrast, serum-type Tf levels showed subtle change in the CSF of iNPH patients after shunt surgery (data not shown). Serum-type Tf influx from the blood to the CSF probably occurs due to passive diffusion, meaning that its concentration before and after surgery does not change. ${ }^{31)}$

We found that neurological clinical grading scales in response to shunt surgery improved after $11.8 \pm 7.7$ months. When correlations between cog- nitive scales and CSF biomarkers were analyzed at various time points, brain-type $\mathrm{Tf}$ levels before shunt surgery showed low $(r=0.351, p=0.042)$ and moderate $(r=0.527, p=0.002)$ correlations with Mini-Mental State Examination (MMSE) and Frontal Assessment Battery (FAB) scores, respectively (Fig. 6A, B). At 3 months post-operative, the increase of brain-type Tf correlated strongly with MMSE scores $(r=0.697, p=0.037)$ and FAB $(r=$ $0.727, p=0.041$ ) scores (Fig. $6 \mathrm{C}$, D; left panel). The increase of brain-type $\mathrm{Tf}$ at post-operative month 12 was moderately correlated with $\operatorname{MMSE}(r=0.549$, 
A

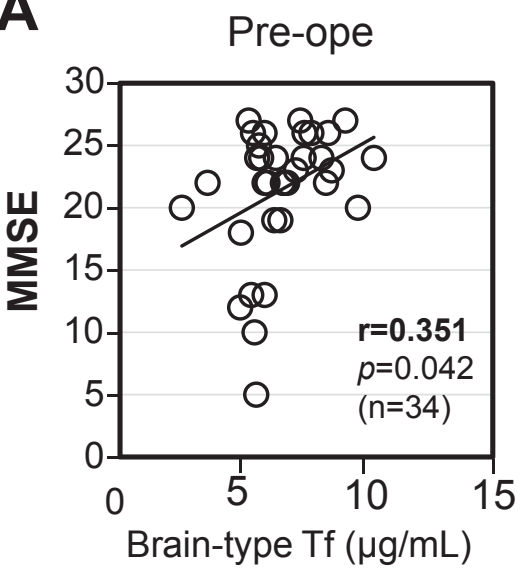

B

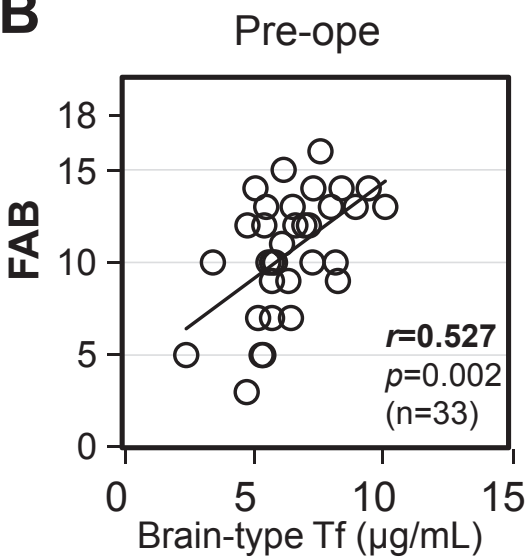

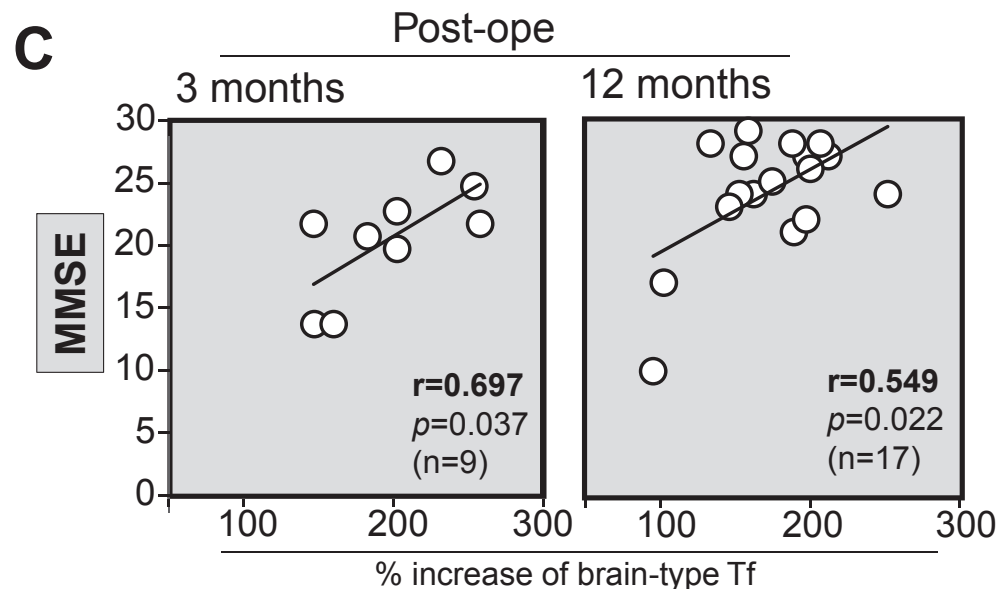

D

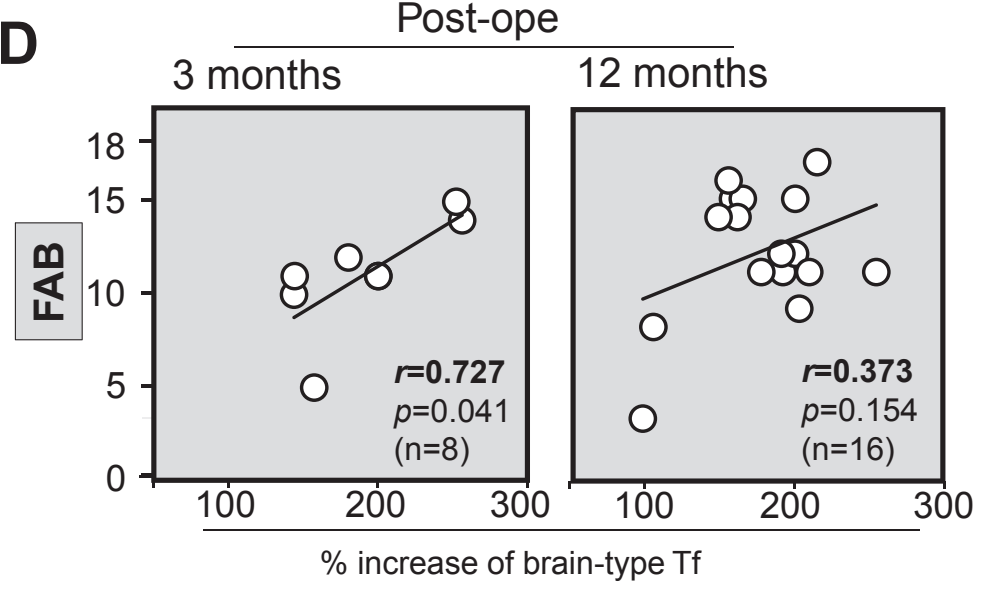

Fig. 6. Correlation between brain-type Tf and cognitive scales. Correlation between pre-operative levels of brain-type Tf and cognitive scales MMSE (A) or FAB (B), are indicated. Brain-type Tf levels before surgery and \% increases at post-operative months 3 and 12 are shown. Correlation between the $\%$ increase and cognitive scales at the post-operative timepoint of $11.8 \pm 7.7$ months is indicated $(\mathrm{C}, \mathrm{D})$.

$p=0.022)$ but not with FAB $(r=0.373, p=0.154)$

(Fig. 6C, D; right panel). Scores for the modified Rankin Scale were not correlated with brain-type Tf levels before or after shunt surgery (data not shown). Furthermore, the increase of sAPP in both the preand post-operative periods was not correlated with MMSE or FAB. Concentration of L-PGDS was not measured due to limitation for CSF specimen available for the analysis. These results suggest that brain-type Tf levels at post-operative month 3 (and 12) predicts recovery from cognitive deficits in iNPH patients. ${ }^{23)}$

Spontaneous intracranial hypotension (SIH). SIH is commonly caused by leakage of the CSF. Patients with SIH often report orthostatic headache, dizziness, hearing disturbance, nausea and vomiting, and cervical pain. Cranial magnetic reso- nance imaging (MRI) of SIH patients revealed cranial hypotension-related manifestations such as diffuse pachymeningeal gadolinium enhancement, sagging of the brain, enlarged pituitary gland, descent of the brain stem, and subdural fluid collection. On radioisotope (RI) scintigraphy, findings include early accumulation of tracer in the bladder and kidneys, and a paucity of activity over the cerebral convexities. Myelography can show the location and extent of a CSF leak. While these abnormal findings are typical for SIH, $20-30 \%$ of SIH patients, however, do not show abnormalities in these clinical tests. ${ }^{32}$ ) We retrospectively studied CSF samples obtained by lumbar puncture of 62 consecutive patients suspected of intracranial hypotension. SIH was diagnosed based on the International Classification of Head Disorders, (3rd edition, beta version, http://beta.ichd-3.org/) 


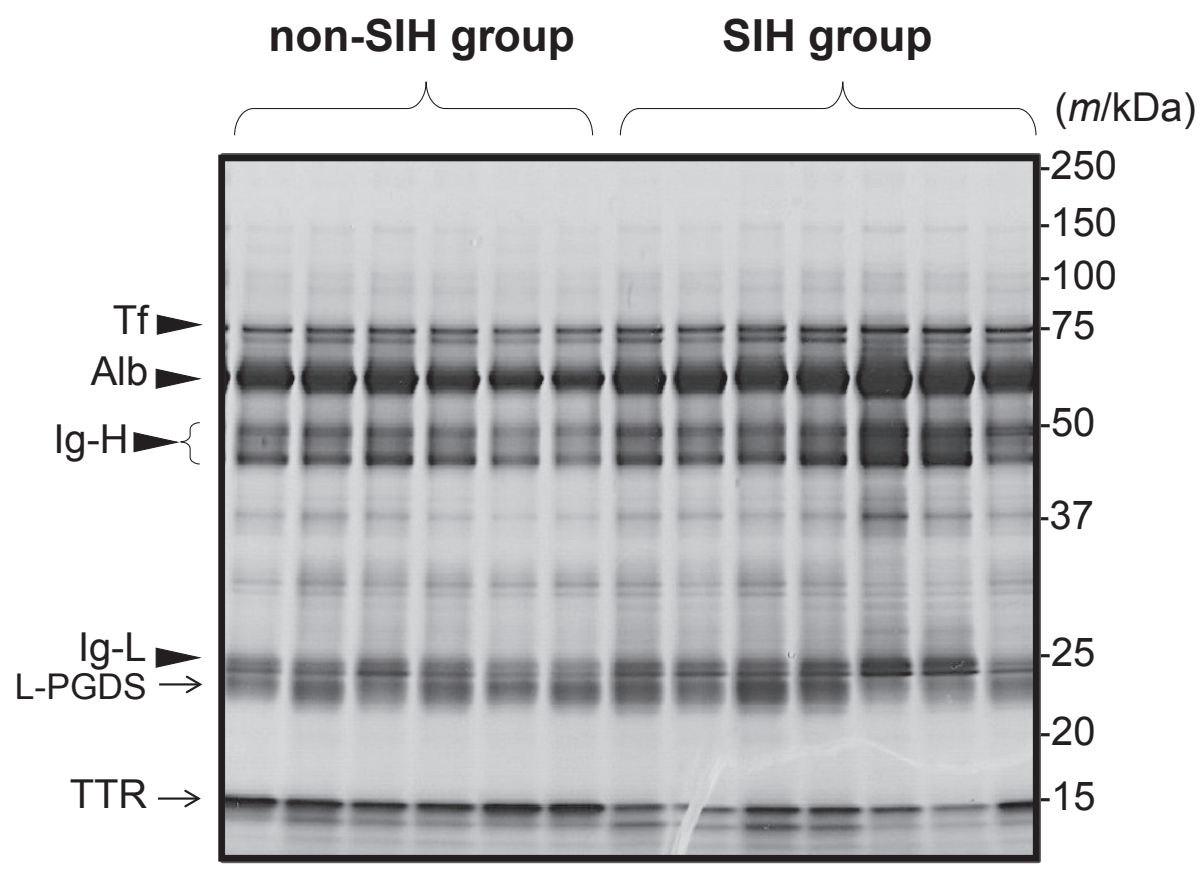

Fig. 7. Human CSF protein pattern for SIH and non-SIH patients. CSF proteins were analyzed by SDS-PAGE followed by silver staining. Arrowheads indicate the positions of serum transferrin (Tf), albumin (Alb), IgG heavy chain (Ig-H) and IgG light chain (Ig-L), respectively. Arrows indicate the position of lipocalin-type prostaglandin D synthase (L-PGDS) and transthyretin (TTR) purified from CSF.

and the diagnostic criteria reported by Schievink et al. ${ }^{33)}$ In this way, 38 patients were classified as $\mathrm{SIH}$ and 24 patients as non-SIH. CSF samples were analyzed based on the groupings, along with the CSF samples of a neurological control group including patients with trigeminal neuralgia or unruptured cerebral artery aneurysm $(\mathrm{n}=10)$.

Most CSF laboratory test results for the SIH and non-SIH groups were within reference intervals. Tests included osmolality, specific gravity, glucose concentration, chloride concentration, $\mathrm{pH}$, and cell counts (leukocytes and erythrocytes). Only CSF protein concentration in the $\mathrm{SIH}$ was significantly higher than that of the non-SIH group $(0.9 \mathrm{mg} / \mathrm{mL}$ versus $0.6 \mathrm{mg} / \mathrm{mL}, p<0.001)$. In contrast, serum protein concentrations were not different between the two groups $(p=0.22)$. In the SIH group, the CSF protein concentration was not correlated with that of serum $(r=0.153, p=0.367)$, suggesting that the increase in CSF protein level was independent of the serum protein levels.

To examine protein composition changes, CSF samples from the SIH and non-SIH patients were subjected to SDS-PAGE followed by silver staining (Fig. 7). Protein patterns were similar between the $\mathrm{SIH}$ and non-SIH groups, and composed principally of serum Tf $(75 \mathrm{kDa})$, albumin $(66 \mathrm{kDa})$, immunoglobulin heavy chain (Ig-H) $(45-50 \mathrm{kDa}$ area), and light chain (Ig-L) $(25 \mathrm{kDa})$, L-PGDS, and TTR. While no apparent differences in protein composition were observed between the two groups, the signal intensity of each protein band, with the exception of the TTR bands, appeared to be increased in the SIH group. We therefore quantified these major proteins together with sAPP, a marker for CSF anomalies.

Albumin, IgG, total Tf, L-PGDS, TTR, sAPP, serum-type Tf and brain-type Tf were quantified by ELISA or Western blotting. Analysis of the data revealed that levels of these proteins, except for TTR, were significantly higher in the SIH than the non-SIH group (Fig. 7). TTR levels in the SIH and non-SIH groups were $24.8 \mu \mathrm{g} / \mathrm{mL}$ and $20.4 \mu \mathrm{g} / \mathrm{mL}$, respectively, $(p=0.054)$. We performed statistical analyses on CSF proteins with a view to their potential use as differentiators (biomarkers) of SIH and non-SIH. Serum-derived proteins such as albumin, IgG, and serum-type transferrin showed differentiation sensitivities and specificities in the ranges of $76.3-78.9 \%$ and $70.8-79.2 \%$, respectively, whereas the sensitivities and specificities of CNS-derived proteins such as L-PGDS, sAPP, and brain-type Tf ranged from $71.1-81.6 \%$ and $50.0-91.7 \%$, respectively. Concen- 
trations of serum albumin, IgG, and Tf were 4070-fold higher than those in the CSF, suggesting that even slight contamination of blood disturbs the accurate estimation of the concentrations of these proteins. Based on these findings, we further analyzed CNS-derived proteins as preferred markers of SIH. Logistic regression analysis revealed odds ratios for L-PGDS, sAPP, and brain-type Tf of 9.15 (95\%CI: $1.15-73.2, p=0.036), 0.64$ (95\%CI: $0.13-$ $3.1, p=0.577)$, and 5.95 (95\%CI: 1.01-35.1, $p=$ $0.049)$, respectively. The combined use of L-PGDS and brain-type $\mathrm{Tf}$ differentiated $\mathrm{SIH}$ from non-SIH with a sensitivity of $94.7 \%$ and specificity of $72.6 \% .^{30}$ )

In $\mathrm{SIH}$, the intracranial pressure decreases due to the leakage of CSF (threshold; less than $60 \mathrm{~mm}$ $\mathrm{H}_{2} \mathrm{O}$ ). Moreover, the concentration of radioisotope (RI) injected into the CSF decreases rapidly and is excreted in the urine. The cutoff for RI residual activity at 24 hours after RI injection is set at $20 \%$. With these clinical tests established to differentiate SIH from non-SIH, we therefore examined correlations between them and CNS-derived protein levels. Significant correlations were observed between the test outcomes and L-PGDS or brain-type Tf. A decrease in RI residual activity was inversely correlated with increases of L-PGDS $(r=-0.56$, $p<0.001)$ and brain-type Tf $(r=-0.50, p<0.001)$. Decreased intracranial pressure was also inversely correlated with increased of L-PGDS $(r=-0.56$, $p<0.001)$ and brain-type Tf $(r=-0.46, p<0.001)$. No other proteins were correlated with intracranial pressure or RI residual activity. ${ }^{30)}$

\section{CSF marker changes in iNPH and SIH}

L-PGDS is mainly produced in the choroid plexus, leptomeninges, and oligodendrocytes, and then secreted into the CSF. ${ }^{26)}$ It is then absorbed in the cranial arachnoid villi and flows into the dural sinuses. ${ }^{26)}$ Mase et al. reported that L-PGDS levels were decreased in (idiopathic) normal pressure hydrocephalus. ${ }^{28)}$ As described above, L-PGDS, brain-type Tf, and sAPP are all decreased in iNPH (Fig. 8B), where CSF production is also suspected to decrease. In contrast, these proteins increase in $\mathrm{SIH}$ (Fig. 8B), while CSF production is suspected to increase to compensate for CSF leakage. L-PGDS, brain-type Tf, and sAPP levels may thus change concomitantly with CSF production.

A possible explanation for the observed upregulation of brain-derived proteins in $\mathrm{SIH}$ patients is that the secretion of these proteins depends on CSF pressure or volume. As such, protein levels decrease in iNPH, in which the brain is compressed by excess CSF. As described above, brain-type Tf and sAPP were normalized or increased after shunt surgery, which bypasses excess CSF from the brain's ventricles to the peritoneal cavity. This normalization suggests that CSF pressure or volume affect the secretion of the brain-derived proteins. Further research is required to clarify the exact molecular mechanisms underlying the increased levels of these proteins.

Blood brain barrier (BBB) and protein permeability. CSF is sequestered from blood by the BBB. As a result, the protein concentration of the CSF is about 100 -fold lower than in the blood. The barrier, however, is leaky, even under normal physiological conditions. Indeed, about $80 \%$ of CSF proteins are derived from the blood, ${ }^{10)}$ with this influx probably due to deficiencies in BBB structures. To this end, pericapillary lamellar processes of astrocytes are not observed in several paraventricular structures such as the posterior pituitary gland (neurohypophysis) and the pineal body. ${ }^{34}$ Under conditions of intracranial hypotension, the influx of different proteins depends on various factors such as their concentration in the blood, their molecular size, and interaction with other molecules. The increase of CSF protein levels in SIH is mainly attributable to albumin, which is biosynthesized exclusively in the liver and then secreted into the blood, suggesting that the increase in albumin is due to enhanced influx from the blood into the CSF. An enhanced inflow is also seen with $\operatorname{Ig} G$, which is mainly produced in the extra-neural lymphatic tissues. Other blood proteins such as serum Tf could flow into the CSF down their concentration gradient. ${ }^{30)}$

\section{Conclusion}

We hypothesize that brain-type Tf secreted from the choroid plexus could be a biomarker of altered CSF production, and as such, useful for diagnosing iNPH. In addition, it may be also applicable to monitoring the normalization of CSF production after shunt surgery and for predicting cognitive recovery. This marker could also be useful for diagnosing SIH, where a marker with high sensitivity and specificity has not been available until now.

\section{Acknowledgments}

This work was supported in part by Japan Agency for Medical Research and Development (AMED) [grant numbers 16hm0102042h0001 and $17 \mathrm{hm0102042h0002}$ and 18hm0102042h0003]; the Japan Science and Technology Agency [grant num- 

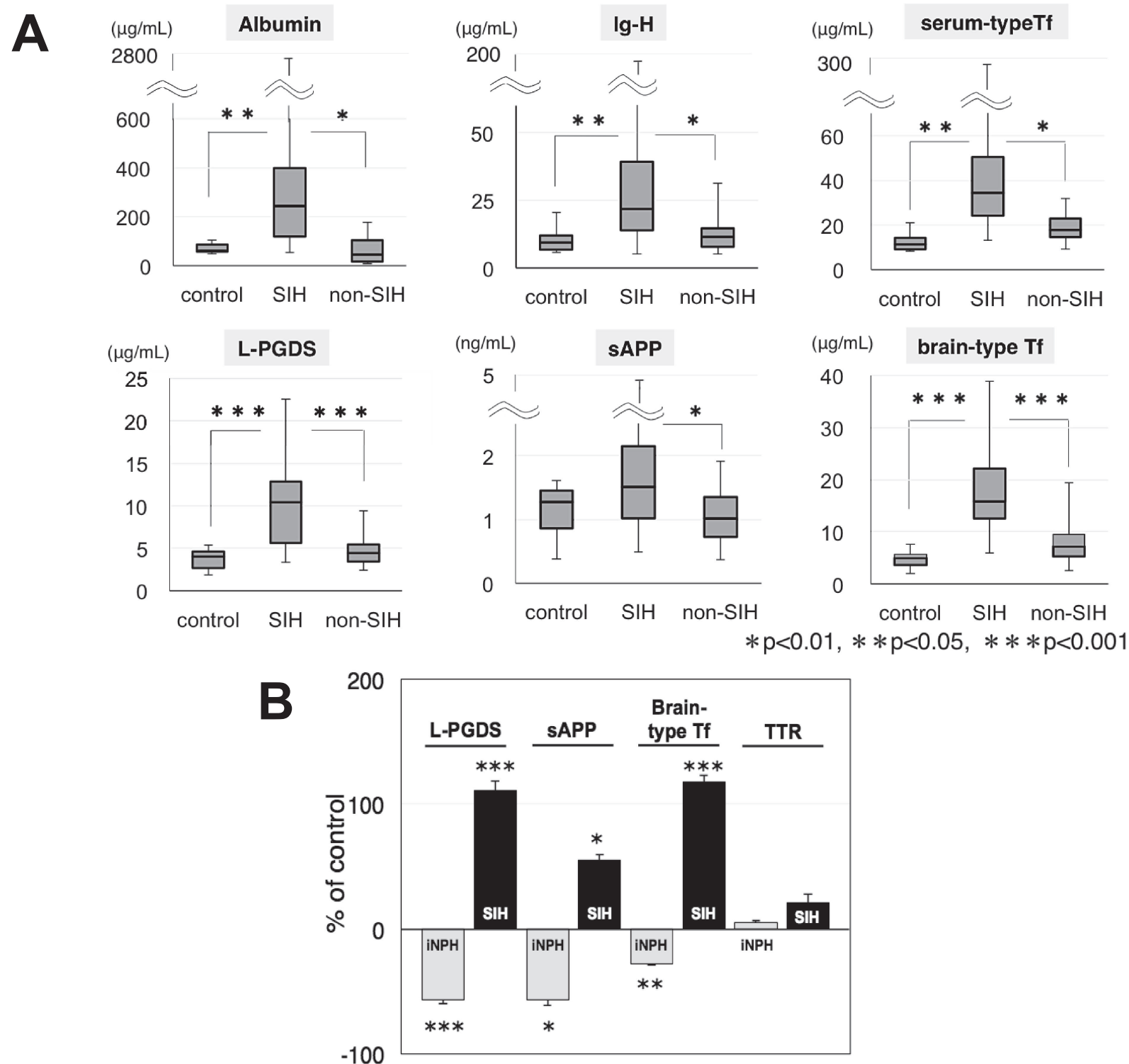

Fig. 8. Box plots of protein concentrations in the CSF of SIH and non-SIH patients. Proteins examined were albumin, IgG heavy chain (Ig-H), serum-type Tf, L-PGDS, sAPP, and brain-type Tf (A). Horizontal lines within boxes show median values; boxes exclude upper and lower interquartile (IQ) ranges; whiskers indicate 1.5 times IQ; ${ }^{*} \mathrm{p}<0.01,{ }^{* *} \mathrm{p}<0.05,{ }^{* * *} \mathrm{p}<0.001$. CSF concentrations of L-PGDS, sAPP, brain-type Tf, and TTR in SIH (black bars) and iNPH (gray bars) are shown in (B); ${ }^{*}$ p $<0.01,{ }^{* *}$ p $<0.05$, $* * * \mathrm{p}<0.001$.

bers AS221Z00232F, AS231Z01053, 241FT0255, ad 149]; the Ministry of Education, Culture, Sports, Science, and Technology of Japan [a Grant-in-Aid for Scientific Research on Innovative Areas, grant number 23110002 (Deciphering sugar chain-based signals regulating integrative neuronal functions), and a Grant-in-Aid for Scientific Research, grant number 23590367]; The General Insurance Association of Japan [a medical grant research on traffic accidents]; and the National Mutual Insurance Federation of Agricultural Cooperatives.

We thank Drs. Hajime Arai and Masakazu Miyajima, Department of Neurosurgery, Juntendo University, for collaboration with the iNPH research, and Drs. Koichi Takahashi and Tatsuo Mima, Department of Neurosurgery, Sanno Hospital, for collaboration with the SIH research. We also acknowledge members of Fukushima Medical Uni- 
versity; Drs. Kyoka Hoshi and Takashi Fuwa, Department of Biochemistry, for biochemical analyses; Dr. Takashi Honda, Department of Human Life Science, for histological analysis; and Dr. Kenneth Nollet, Department of Blood Transfusion and Transplantation Immunology, for proofreading the manuscript.

\section{Contributors}

All authors equally contributed to all parts of the review. All authors revised the manuscript and approved the final version.

\section{References}

1) Koeppen, A.H. (1995) The history of iron in the brain. J. Neurol. Sci. 134 (Suppl.), 1-9.

2) Ward, R.J., Zucca, F.A., Duyn, J.H., Crichton, R.R. and Zecca, L. (2014) The role of iron in brain ageing and neurodegenerative disorders. Lancet Neurol. 13, 1045-1060.

3) Andrews, N.C. (2000) Iron homeostasis: Insights from genetics and animal models. Nat. Rev. Genet. 1, 208-217.

4) Zecca, L., Youdim, M.B., Riederer, P., Connor, J.R. and Crichton, R.R. (2004) Iron, brain ageing and neurodegenerative disorders. Nat. Rev. Neurosci. 5, 863-873.

5) de Jong, G., van Dijk, J.P. and van Eijk, H.G. (1990) The biology of transferrin. Clin. Chim. Acta 190, $1-46$.

6) Kurokawa, H., Mikami, B. and Hirose, M. (1995) Crystal structure of diferric hen ovotransferrin at 2.4 Å resolution. J. Mol. Biol. 254, 196-207.

7) Rouault, T.A. and Cooperman, S. (2006) Brain iron metabolism. Semin. Pediatr. Neurol. 13, 142-148.

8) Blay, P., Nilsson, C., Hansson, S., Owman, C., Aldred, A.R. and Schreiber, G. (1994) An in vivo study of the effect of 5-HT and sympathetic nerves on transferrin and transthyretin mRNA expression in rat choroid plexus and meninges. Brain Res. 662, 148-154.

9) De Arriba Zerpa, G.A., Saleh, M.A.-C., Fernandez, P.M., Guillou, F., Espinosa De Los Monteros, A., De Vellis, J. et al. (2000) Alternative splicing prevents transferrin secretion during differentiation of a human oligodendrocyte cell line. J. Neurosci. Res. 61, 388-395.

10) Reiber, H. and Peter, J.B. (2001) Cerebrospinal fluid analysis: Disease-related data patterns and evaluation programs. J. Neurol. Sci. 184, 101-122.

11) Hoffmann, A., Nimtz, M., Getzlaff, R. and Conradt, H.S. (1995) 'Brain-type' N-glycosylation of asialotransferrin from human cerebrospinal fluid. FEBS Lett. 359, 164-168.

12) Futakawa, S., Nara, K., Miyajima, M., Kuno, A., Ito, H., Kaji, H. et al. (2012) A unique N-glycan on human transferrin in CSF: A possible biomarker for iNPH. Neurobiol. Aging 33, 1807-1815.
13) Kizuka, Y., Kitazume, S., Fujinawa, R., Saito, T., Iwata, N., Saido, T.C. et al. (2015) An aberrant sugar modification of BACE1 blocks its lysosomal targeting in Alzheimer's disease. EMBO Mol. Med. 7, 175-189.

14) Fagerberg, L., Hallstrom, B.M., Oksvold, P., Kampf, C., Djureinovic, D., Odeberg, J. et al. (2014) Analysis of the human tissue-specific expression by genome-wide integration of transcriptomics and antibody-based proteomics. Mol. Cell Proteomics 13, 397-406.

15) Nakakita, S., Natsuka, S., Ikenaka, K. and Hase, S. (1998) Development-dependent expression of complex-type sugar chains specific to mouse brain. J. Biochem. 123, 1164-1168.

16) Hoffmann, A., Nimtz, M., Wurster, U. and Conradt, H.S. (1994) Carbohydrate structures of $\beta$-trace protein from human cerebrospinal fluid: Evidence for "brain-type" $N$-glycosylation. J. Neurochem. 63, 2185-2196.

17) Wyss, D.F., Choi, J.S., Li, J., Knoppers, M.H., Willis, K.J., Arulanandam, A.R. et al. (1995) Conformation and function of the N-linked glycan in the adhesion domain of human CD2. Science 269, 1273-1278.

18) Nagae, M., Morita-Matsumoto, K., Arai, S., Wada, I., Matsumoto, Y., Saito, K. et al. (2014) Structural change of $N$-glycan exposes hydrophobic surface of human transferrin. Glycobiology 24, 693-702.

19) Noinaj, N., Easley, N.C., Oke, M., Mizuno, N., Gumbart, J., Boura, E. et al. (2012) Structural basis for iron piracy by pathogenic Neisseria. Nature 483, 53-58.

20) Stryer, L. (1965) The interaction of a naphthalene dye with apomyoglobin and apohemoglobin. A fluorescent probe of non-polar binding sites. J. Mol. Biol. 13, 482-495.

21) Greene, M.F., Benacerraf, B. and Crawford, J.M. (1985) Hydranencephaly: US appearance during in utero evolution. Radiology 156, 779-780.

22) Pavone, P., Pratico, A.D., Vitaliti, G., Ruggieri, M., Rizzo, R., Parano, E. et al. (2014) Hydranencephaly: Cerebral spinal fluid instead of cerebral mantles. Ital. J. Pediatr. 40, 79.

23) Murakami, Y., Matsumoto, Y., Hoshi, K., Ito, H., Fuwa, T.J., Yamaguchi, Y. et al. (2018) Rapid increase of 'brain-type' transferrin in cerebrospinal fluid after shunt surgery for idiopathic normal pressure hydrocephalus: A prognosis marker for cognitive recovery. J. Biochem. 164, 205-213.

24) Kochibe, N. and Matta, K.L. (1989) Purification and properties of an $N$-acetylglucosamine-specific lectin from Psathyrella velutina mushroom. J. Biol. Chem. 264, 173-177.

25) Silverberg, G.D., Mayo, M., Saul, T., Rubenstein, E. and McGuire, D. (2003) Alzheimer's disease, normal-pressure hydrocephalus, and senescent changes in CSF circulatory physiology: A hypothesis. Lancet Neurol. 2, 506-511.

26) Beuckmann, C.T., Lazarus, M., Gerashchenko, D., Mizoguchi, A., Nomura, S., Mohri, I. et al. (2000) 
Cellular localization of lipocalin-type prostaglandin $\mathrm{D}$ synthase ( $\beta$-trace) in the central nervous system of the adult rat. J. Comp. Neurol. 428, 6278.

27) Kamenetz, F., Tomita, T., Hsieh, H., Seabrook, G., Borchelt, D., Iwatsubo, T. et al. (2003) APP processing and synaptic function. Neuron 37, 925937.

28) Mase, M., Yamada, K., Shimazu, N., Seiki, K., Oda, H., Nakau, H. et al. (2003) Lipocalin-type prostaglandin D synthase ( $\beta$-trace) in cerebrospinal fluid: A useful marker for the diagnosis of normal pressure hydrocephalus. Neurosci. Res. 47, 455459.

29) Miyajima, M., Nakajima, M., Ogino, I., Miyata, H., Motoi, Y. and Arai, H. (2013) Soluble amyloid precursor protein $\alpha$ in the cerebrospinal fluid as a diagnostic and prognostic biomarker for idiopathic normal pressure hydrocephalus. Eur. J. Neurol. 20, 236-242.

30) Murakami, Y., Takahashi, K., Hoshi, K., Ito, H. Kanno, M., Saito, K. et al. (2018) Spontaneous intracranial hypotension is diagnosed by a combi- nation of lipocalin-type prostaglandin D synthase and brain-type transferrin in cerebrospinal fluid. Biochim. Biophys. Acta, Gen. Subj. 1862, 18351842.

31) Hoshi, K., Matsumoto, Y., Ito, H., Saito, K., Honda, T., Yamaguchi, Y. et al. (2017) A unique glycanisoform of transferrin in cerebrospinal fluid: A potential diagnostic marker for neurological diseases. Biochim. Biophys. Acta 1861, 2473-2478.

32) Schievink, W.I. (2006) Spontaneous spinal cerebrospinal fluid leaks and intracranial hypotension. JAMA 295, 2286-2296.

33) Schievink, W.I. and Maya, M.M. (2011) Frequency of intracranial aneurysms in patients with spontaneous intracranial hypotension. J. Neurosurg. 115, $113-115$

34) Malcolm, B. and Carpenter, S.J. (1983) Human Neuroanatomy, 8th edition. Williams \& Wilkins, Baltimore, MD.

(Received Dec. 3, 2018; accepted Mar. 26, 2019)

\section{Profile}

Yuta Murakami was born in Aomori Prefecture in 1984 and graduated from Fukushima Medical University School of Medicine in 2011. After graduation and a two-year clinical training, he entered Fukushima Medical University Graduate School of Medicine and directed by Prof. Kiyoshi Saito, Department of Neurosurgery and Prof. Yasuhiro Hashimoto, Department of Biochemitsry. He is engaged in glycan biomarkers in cerebrospinal fluid for diagnosing neurological diseases. In 2019, he became an assistant professor of Department of Neurosurgery, Fukushima Medical University School of Medicine.

\section{Profile}

Yasuhiro Hashimoto was born in Fukushima Prefecture in 1954 and graduated from Fukushima Medical University School of Medicine in 1979. After graduation he entered The University of Tokyo Graduate School of Medicine and directed by Prof. Tamio Yamakawa, Department of Biochemistry. In 1983, he was appointed to be an investigator of Tokyo Metropolitan Institute of Medical Science. He was a visiting associated professor, Department of Biochemistry, University of Massachusetts Medical Center between 1988 and 1992. He was appointed to be a team leader of Glyco-chain Functions Laboratory, Frontier Research System, RIKEN Institute in 1999. He found that Alzheimer's beta-secretase, BACE1, which triggers amyloid pathology, cleaves glycosyltransferases and controls glycan expression. This was the first report for

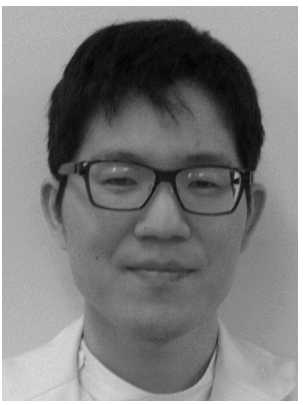
physiological function of $\beta$-secretase. In 2007, he became a professor of Department of Biochemistry, Fukushima Medical University School of Medicine. He has studied on glycan biomarkers in cerebrospinal fluid for diagnosing neurological diseases.

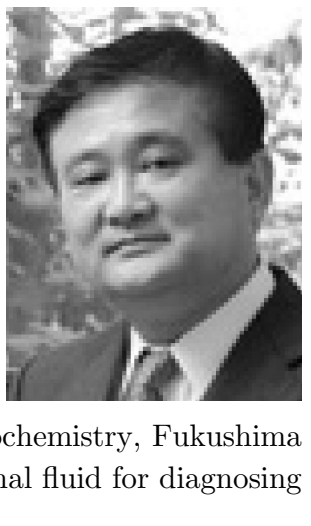

